\title{
A multiscale Molecular Dynamics approach to Contact Mechanics
}

\author{
C. Yang, U. Tartaglino* and B.N.J. Persson \\ IFF, FZ-Jülich, 52425 Jülich, Germany
}

\begin{abstract}
The friction and adhesion between elastic bodies are strongly influenced by the roughness of the surfaces in contact. Here we develop a multiscale molecular dynamics approach to contact mechanics, which can be used also when the surfaces have roughness on many different length-scales, e.g., for self affine fractal surfaces. As an illustration we consider the contact between randomly rough surfaces, and show that the contact area varies linearly with the load for small load. We also analyze the contact morphology and the pressure distribution at different magnification, both with and without adhesion. The calculations are compared with analytical contact mechanics models based on continuum mechanics.
\end{abstract}

\section{Introduction}

Adhesion and friction between solid surfaces are common phenomenons in nature and of extreme importance in biology and technology. Most surfaces of solids have roughness on many different length scales 1, 2], and it is usually necessary to consider many decades in length scale when describing the contact between solids 3 . This makes it very hard to describe accurately the contact mechanics between macroscopic solids using computer simulation methods, e.g., atomistic molecular dynamics, or finite element calculations based on continuum mechanics.

Consider a solid with a nominally flat surface. Let $x, y, z$ be a coordinate system with the $x, y$ plane parallel to the surface plane. Assume that $z=h(\mathbf{x})$ describe the surface height profile, where $\mathbf{x}=(x, y)$ is the position vector within the surface plane. The most important property characterizing a randomly rough surface is the surface roughness power spectrum $C(\mathbf{q})$ defined by $[3,4]$

$$
C(\mathbf{q})=\frac{1}{(2 \pi)^{2}} \int d^{2} x\langle h(\mathbf{x}) h(\mathbf{0})\rangle e^{i \mathbf{q} \cdot \mathbf{x}} .
$$

Here $\langle\ldots\rangle$ stands for ensemble average and we have assumed that $h(\mathbf{x})$ is measured from the average surface plane so that $\langle h\rangle=0$. In what follows we will assume that the statistical properties of the surface are isotropic, in which case $C(q)$ will only depend on the magnitude $q=|\mathbf{q}|$ of the wave vector $\mathbf{q}$.

Many surfaces tend to be nearly self-affine fractal. A self-affine fractal surface has the property that if part of the surface is magnified, with a magnification which in general is appropriately different in the perpendicular direction to the surface as compared to the lateral directions, then the surface "looks the same", i.e., the statistical properties of the surface are invariant under the scale transformation [3]. For a self-affine surface the power spectrum has the power-law behavior

$$
C(q) \sim q^{-2(H+1)},
$$

\footnotetext{
${ }^{*}$ Present address: DEMOCRITOS National Simulation Center, Via Beirut 2-4, I-34014 Trieste, Italy; e-mail: tartagli@sissa.it
}

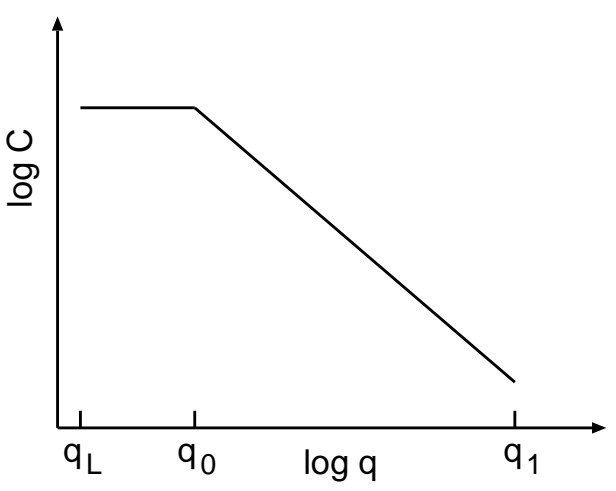

FIG. 1: Surface roughness power spectrum of a surface which is self affine fractal for $q_{1}>q>q_{0}$. The long-distance rolloff wave vector $q_{0}$ and the short distance cut-off wave vector $q_{1}$ depend on the system under consideration. The slope of the $\log C-\log q$ relation for $q>q_{0}$ determines the fractal exponent of the surface. The lateral size $L$ of the surface (or of the studied surface region) determines the smallest possible wave vector $q_{L}=2 \pi / L$.

where the Hurst exponent $H$ is related to the fractal dimension $D_{\mathrm{f}}$ of the surface via $H=3-D_{\mathrm{f}}$. Of course, for real surfaces this relation only holds in some finite wave vector region $q_{0}<q<q_{1}$, and in a typical case $C(q)$ has the form shown in Fig. 1. Note that in many cases there is a roll-off wavevector $q_{0}$ below which $C(q)$ is approximately constant.

Let us consider the contact between an elastic solid with a flat surface and a hard randomly rough substrate. Fig. 2 shows the contact between the solids at increasing magnification $\zeta$. At low magnification $(\zeta=1)$ it looks as if complete contact occurs between the solids at many macro asperity contact regions, but when the magnification is increased smaller length scale roughness is detected, and it is observed that only partial contact occurs at the asperities. In fact, if there would be no short distance cut-off the true contact area would vanish. In reality, however, a short distance cut-off will always exist since the shortest possible length is an atomic distance. In many cases the local pressure at asperity contact regions at high magnification will become so high that the 
material yields plastically before reaching the atomic dimension. In these cases the size of the real contact area will be determined mainly by the yield stress of the solid.

The magnification $\zeta$ refers to some (arbitrary) chosen reference length scale. This could be, e.g., the lateral size $L$ of the nominal contact area in which case $\zeta=L / \lambda$, where $\lambda$ is the shortest wavelength roughness which can be resolved at magnification $\zeta$. In this paper we will instead use the roll-off wavelength $\lambda_{0}=2 \pi / q_{0}$ as the reference length so that $\zeta=\lambda_{0} / \lambda$.

Recently, a very general contact mechanics theory has been developed which can be applied to both stationary and sliding contact for viscoelastic solids (which includes elastic solids as a special case) [4]. The theory was originally developed in order to describe rubber friction on rough substrates. For elastic solids the theory can also be applied when the adhesional interaction is taken into account [5]. In contrast to earlier contact mechanics theories, the theory presented in Ref. [4, 5] is particularly accurate close to complete contact, as would be the case for, e.g., rubber on smooth surfaces. The basic idea behind the theory is to study the contact at different magnification. In particular, the theory describes the change in the stress distribution $P(\sigma, \zeta)$ as the magnifications $\zeta$ increases. Here

$$
P(\sigma, \zeta)=\langle\delta(\sigma-\sigma(\mathbf{x}, \zeta))\rangle
$$

is the stress distribution at the interface when the surface roughness with wavelength smaller than $\lambda=\lambda_{0} / \zeta$ has been removed. In (2), $\langle\ldots\rangle$ stands for ensemble average, and $\sigma(\mathbf{x}, \zeta)$ is the perpendicular stress at the interface when surface roughness with wavelength shorter than $\lambda=\lambda_{0} / \zeta$ has been removed. It is clear that as the magnification $\zeta$ increases, the distribution $P(\sigma, \zeta)$ will be broader and broader and the theory describes this in detail. The (normalized) area of real contact (projected on the $x y$-plane) at the magnification $\zeta$ can be written as

$$
\frac{A(\zeta)}{A_{0}}=\int_{0^{+}}^{\infty} d \sigma P(\sigma, \zeta)
$$

where the lower integration limit $0^{+}$indicate that the delta function at the origin $\sigma=0$ (arising from the noncontact area) should be excluded from the integral. The rubber friction theory described in Ref. [4] depends on the function $A(\zeta) / A_{0}$ for all magnifications. This just reflects the fact that the friction force results from the viscoelastic deformations of the rubber on all length scales, and when evaluating the contribution to the friction from the viscoelastic deformations on the length scale $\lambda$, it is necessary to know the contact between the rubber and the substrate at the magnification $\zeta=\lambda_{0} / \lambda$. Thus, not just the area of real (atomic) contact is of great interest, but many important applications require the whole function $A(\zeta)$, and the pressure distribution $P(\sigma, \zeta)$.

In order to accurately reproduce the contact mechanics between elastic solids, it is in general necessary to

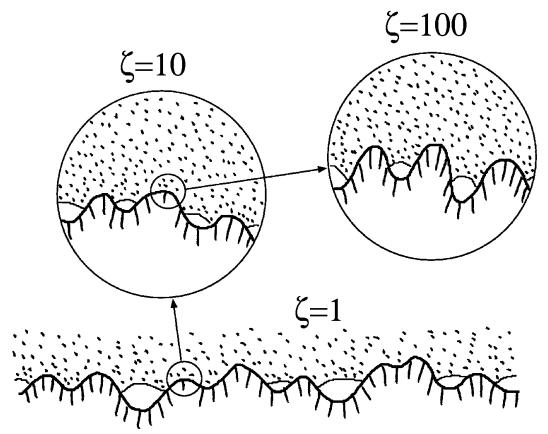

FIG. 2: A rubber block (dotted area) in adhesive contact with a hard rough substrate (dashed area). The substrate has roughness on many different length scales and the rubber makes partial contact with the substrate on all length scales. When a contact area is studied at low magnification $(\zeta=1)$ it appears as if complete contact occurs in the macro asperity contact regions, but when the magnification is increased it is observed that in reality only partial contact occurs.

consider solid blocks which extend a similar distance in the direction normal to the nominal contact area as the linear size of the contact area. This leads to an enormous number of atoms or dynamical variables already for relatively small systems. In this paper we develop a multiscale approach to contact mechanics where the number of dynamical variables scales like $\sim N^{2}$ rather than as $\sim N^{3}$, where $N \times N$ is the number of atoms in the nominal contact area. As application we consider the contact mechanics between randomly rough surfaces both with and without adhesion, and compare the results with analytical contact mechanics theories.

\section{Multiscale molecular dynamics}

Let us discuss the minimum block-size necessary in a computer simulation for an accurate description of the contact mechanics between two semi-infinite elastic solids with nominal flat surfaces. Assume that the surface roughness power spectrum has a roll-off wavevector $q=$ $q_{0}$ corresponding to the roll-off wavelength $\lambda_{0}=2 \pi / q_{0}$. In this case the minimum block must extend $L_{x} \approx \lambda_{0}$ and $L_{y} \approx \lambda_{0}$ along the $x$ and $y$-directions. Furthermore, the block must extend at least a distance $L_{z} \approx \lambda_{0}$ in the direction perpendicular to the nominal contact area. The latter follows from the fact that a periodic stress distribution with wavelength $\lambda$ acting on the surface of a semi-infinite elastic solid gives rise to a deformation field which extends a distance $\sim \lambda$ into the solid. Thus, the minimum block is a cube with the side $L=\lambda_{0}$.

As an example, if $\lambda_{0}$ corresponds to 1000 atomic spacings, one must at least consider a block with $1000 \times 1000$ atoms within the $x y$-contact plane, i.e., one would need to study the elastic deformation in a cubic block with at least $10^{9}$ atoms. However, it is possible to drastically reduce the number of dynamical variables without 
(a)

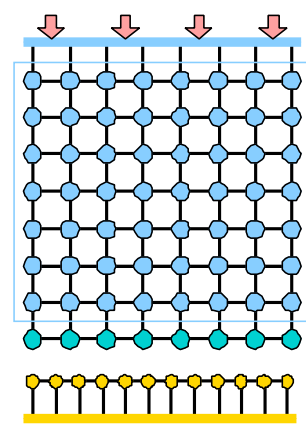

(b)

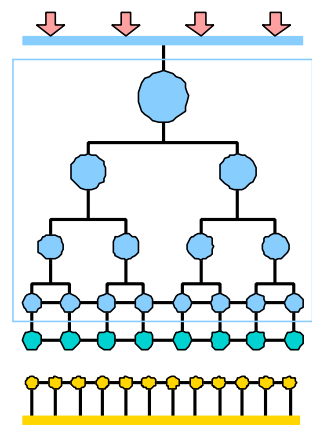

FIG. 3: Schematic structure of the model. (a) The fully atomistic model. (b) The multiscale smartblock model, where the solid in (a) is coarse grained by replacing groups of atoms with bigger "atoms".

loss of accuracy if one notes that an interfacial roughness with wavelength $\lambda$ will give rise to a deformation field in the block which extends a distance $\lambda$ into the solid, and which varies spatially over a typical length scale $\lambda$. Thus when we study the deformation a distance $z$ into the block we do not need to describe the solid on the atomistic level, but we can coarse-grain the solid by replacing groups of atoms with bigger "atoms" as indicated schematically in Fig. 3 If there are $N \times N$ atoms in the nominal contact area one need $n \approx \ln N$ "atomic" layers in the $z$-direction. Moreover the number of atoms in each layer decreases in a geometric progression every time the coarse graining procedure is applied, so that the total number of particles is of order $N^{2}$ instead of $N^{3}$. This results in a huge reduction in the computation time for large systems. This multiscale approach may be implemented in various ways, and in the Appendix A we outline the procedure we have used in this paper which we refer to as the smartblock. Another implementation similar to our approach can be found in Ref. [6].

The smartblock model should accurately describe the deformations in the solids as long as the deformations varies slowly enough with time. However, the model cannot accurately describe the propagation of short wavelength phonons. This is, in fact, true with all forms of Hamiltonian multiscale descriptions of solids, because of the energy conservation together and the unavoidable loss of information in the coarse grained region. In principle it should be possible to prevent the back reflection of short wavelength phonons by describing the coarse grained region as a continuum, where the numerical calculation can be carried on through a Finite Element scheme. [7, [8, 9, 10] This indeed would require no coarse graining at all in the region treated with molecular dynamics, and a proper choice of the matching conditions between the atomistic and the continuum region. However, with respect to contact mechanics and adhesion the back reflection of short wavelength phonons is not an im-

portant limitation. With respect to sliding friction it may be a more severe limitation in some cases.

Figure 3 illustrates a case where the block is in the form of a cube with atomically flat surfaces. It is possible to obtain curved surfaces of nearly arbitrary shape by "gluing" the upper surface of the block to a hard curved surface profile. This was described in detail in Ref. [4]. The elastic modulus and the shear modulus of the solid can be fixed at any value by proper choice of the elongation and bending spring constants for the springs between the atoms (see Ref. 4] and Appendix A). The upper surface of the smartblock can be moved with arbitrary velocity in any direction, or an external force of arbitrary magnitude can be applied to the upper surface of the smartblock. We have also studied sliding friction problems where the upper surface of the smartblock is connected to a spring which is pulled in some prescribed way. The computer code also allows for various lubricant fluids between the solid surfaces of the block and the substrate. Thus the present model is extremely flexible and can be used to study many interesting adhesion and friction phenomena, which we will report on elsewhere.

We note that with respect to contact mechanics, when the slopes of the surfaces are small, i.e. when the surfaces are almost horizontal, one of the two surfaces can be considered flat, while the profile of the other surface has to be replaced by the difference of the two original profiles [11. Thus, if the substrate has the profile $z=h_{1}(\mathbf{x})$ and the block has the profile $z=h_{2}(\mathbf{x})$, then we can replace the actual system with a fictive one where the block has an atomically smooth surface while the substrate profile $h(\mathbf{x})=h_{2}(\mathbf{x})-h_{1}(\mathbf{x})$. Furthermore, if the original solids have the elastic modulus $E_{1}$ and $E_{2}$, and the Poisson ratio $\nu_{1}$ and $\nu_{2}$, then the substrate in the fictive system can be treated as rigid and the block as elastic with the elastic modulus $E$ and Poisson ratio $\nu$ chosen so that $\left(1-\nu^{2}\right) / E=\left(1-\nu_{1}^{2}\right) / E_{1}+\left(1-\nu_{2}^{2}\right) / E_{2}$.

The results presented below have been obtained for a rigid and rough substrate. The atoms in the bottom layer of the block form a simple square lattice with lattice constant $a$. The lateral dimensions $L_{x}=N_{x} a$ and $L_{y}=$ $N_{y} a$. For the block, $N_{x}=400$ and $N_{y}=400$. Periodic boundary conditions are applied in the $x y$ plane. The lateral size of the block is equal to that of substrate, but we use different lattice constant $b \approx a / \phi$, where $\phi=$ $(1+\sqrt{5}) / 2$ is the golden mean, in order to avoid the formation of commensurate structures at the interface. The mass of a block atom is 197 a.m.u. and the lattice constant of the block is $a=2.6 \AA$, reproducing the atomic mass and the density of gold. We consider solid blocks with two different Young's moduli: a hard solid with $E=77 \mathrm{GPa}$, like in gold, and a soft one with $0.5 \mathrm{GPa}$. The corresponding shear moduli were $G=27 \mathrm{GPa}$ and $0.18 \mathrm{GPa}$, respectively.

The atoms at the interface between the block and the 
substrate interact with the potential

$$
U(r)=4 \epsilon\left[\left(\frac{r_{0}}{r}\right)^{12}-\alpha\left(\frac{r_{0}}{r}\right)^{6}\right]
$$

where $r$ is the distance between a pair of atoms. When $\alpha=1$, Eq. (4) is the standard Lennard-Jones potential. The parameter $\epsilon$ is the binding energy between two atoms at the separation $r=2^{1 / 6} r_{0}$. When we study contact mechanics without adhesion we put $\alpha=0$. In the calculations presented below we have used $r_{0}=3.28 \AA$ and $\epsilon=18.6 \mathrm{meV}$, which (when $\alpha=1$ ) gives an interfacial binding energy (per unit area) 12$] \Delta \gamma \approx 4 \epsilon / a^{2} \approx$ $11 \mathrm{meV} / \AA^{2}$.

\section{Self affine fractal surfaces}

In our calculations we have used self affine fractal surfaces generated as outlined in Ref. 3]. Thus, the surface height is written as

$$
h(\mathbf{x})=\sum_{\mathbf{q}} B(\mathbf{q}) e^{i[\mathbf{q} \cdot \mathbf{x}+\phi(\mathbf{q})]}
$$

where, since $h(\mathbf{x})$ is real, $B(-\mathbf{q})=B(\mathbf{q})$ and $\phi(-\mathbf{q})=$ $-\phi(\mathbf{q})$. If $\phi(\mathbf{q})$ are independent random variables, uniformly distributed in the interval $[0,2 \pi[$, then one can easily show that higher order correlation functions involving $h(\mathbf{x})$ can be decomposed into a product of pair correlations, which implies that the height probability distribution $P_{h}=\langle\delta(h-h(\mathbf{x}))\rangle$ is Gaussian 3]. However, such surfaces can have arbitrary surface roughness power spectrum. To prove this, substitute (5) into (1) and use that

$$
\left\langle e^{i \phi\left(\mathbf{q}^{\prime}\right)} e^{i \phi\left(\mathbf{q}^{\prime \prime}\right)}\right\rangle=\delta_{\mathbf{q}^{\prime},-\mathbf{q}^{\prime \prime}}
$$

gives

$$
\begin{gathered}
C(\mathbf{q})=\frac{1}{(2 \pi)^{2}} \int d^{2} x \sum_{\mathbf{q}^{\prime}}\left|B\left(\mathbf{q}^{\prime}\right)\right|^{2} e^{i\left(\mathbf{q}-\mathbf{q}^{\prime}\right) \cdot \mathbf{x}} \\
=\sum_{\mathbf{q}^{\prime}}\left|B\left(\mathbf{q}^{\prime}\right)\right|^{2} \delta\left(\mathbf{q}-\mathbf{q}^{\prime}\right)
\end{gathered}
$$

Replacing

$$
\sum_{\mathbf{q}} \rightarrow \frac{A_{0}}{(2 \pi)^{2}} \int d^{2} q,
$$

where $A_{0}$ is the nominal surface area, gives

$$
C(\mathbf{q})=\frac{A_{0}}{(2 \pi)^{2}}|B(\mathbf{q})|^{2} .
$$

Thus, if we choose

$$
B(\mathbf{q})=(2 \pi / L)[C(\mathbf{q})]^{1 / 2},
$$

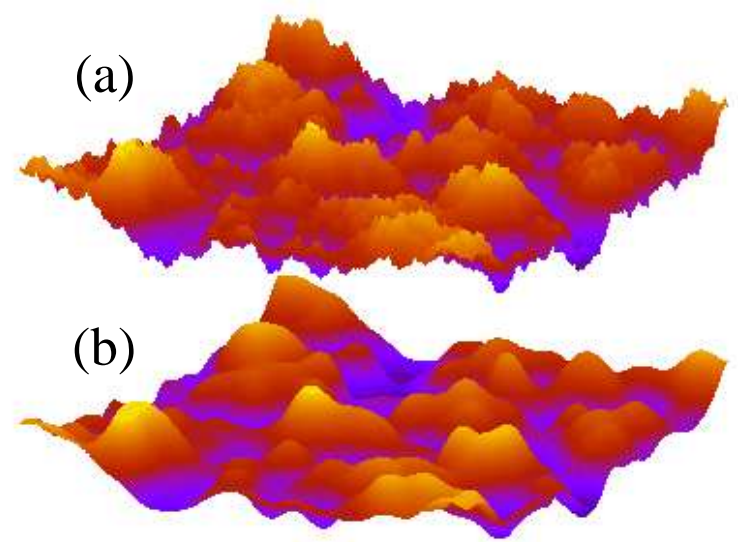

FIG. 4: (a) Fractal surface with the large wavevector cut-off $q_{1}=2 \pi / b \approx 216 q_{0}$. (b) The same surface as in (a) but at lower resolution with $q_{1}=4 q_{0}$. For a square $1040 \AA \times 1040 \AA$ surface area. The fractal dimension $D_{\mathrm{f}}=2.2$ and the rootmean-square roughness amplitude is $10 \AA$.

where $L=A_{0}^{1 / 2}$, then the surface roughness profile (5) has the surface roughness power density $C(\mathbf{q})$. If we assume that the statistical properties of the rough surface are isotropic, then $C(\mathbf{q})=C(q)$ is a function of the magnitude $q=|\mathbf{q}|$, but not of the direction of $\mathbf{q}$. The randomly rough substrate surfaces used in our numerical calculations where generated using (5) and (6) and assuming that the surface roughness power spectra have the form shown in Fig. 1 with the fractal dimension $D_{\mathrm{f}}=2.2$ and the roll-off wavevector $q_{0}=3 q_{L}$, where $q_{L}=2 \pi / L_{x}$. We have chosen $q_{0}=3 q_{L}$ rather than $q_{0}=q_{L}$ since the former value gives some self-averaging and less noisy numerical results. We also used $q_{1}=2 \pi / b \approx 216 q_{0}$ (topography (a) in Fig. (4) and some surfaces with several smaller values for $q_{1}$ (Fig. 4 (b) shows the topography when $q_{1}=4 q_{0}$ ), corresponding to lower magnification (see Sec. 4).

\section{Numerical results}

In this section we illustrate our multiscale molecular dynamics (MD) approach by some applications. We first compare the MD results to two known contact mechanics results from continuum mechanics. Next we discuss contact mechanics for randomly rough surfaces both with and without adhesion.

\subsection{Test cases: Hertz contact and complete} contact

In 1881 Hertz presented an exact solution for the contact between two perfectly elastic solids with local quadratic profiles. The results were derived using the elastic continuum model and neglecting the adhesion be- 
tween the solids. In addition, Hertz assumed that the interfacial friction vanishes so that no shear stress can develop at the interface between the solids. When a spherical asperity is squeezed against a flat surface a circular contact area (radius $r_{\mathrm{H}}$ ) is formed, where the pressure decreases continuously from the center $r=0$ to the periphery $r=r_{\mathrm{H}}$ of the contact according to

$$
\sigma=\sigma_{\mathrm{H}}\left[1-\left(\frac{r}{r_{\mathrm{H}}}\right)^{2}\right]^{1 / 2} .
$$

Let us compare the prediction of our atomistic model with the Hertz theory. We use the Lennard-Jones potential with $\alpha=0$, i.e. without the attractive term. In Fig. 5 we compare the Hertz contact pressure (green line) with our numerical data (red data points). The numerical data were obtained for a rigid spherical tip squeezed against a flat elastic surface. Note that the pressure obtained from the MD calculation has a tail beyond the Hertz contact radius $r_{\mathrm{H}}$. Similar "pressure tails" were recently observed in molecular dynamics simulations by Luan and Robbins [13]. The tail reflects the non-zero extent of the atom-atom interaction potential. The deviation between the molecular dynamics results and the continuum mechanics results should decrease continuously as the size of the system increases.

At the atomic level there is no unique way to define when two solids are in contact, and one may use several different criteria. One method is based on the force acting between the atoms at the interface and works best when the adhesional interaction is neglected. Thus, when two surfaces approach each other, the repulsive force between the atoms increases. We may define contact when the repulsive force is above some critical value. When adhesion is included the interaction between the wall atoms becomes more long-ranged and it is less obvious how to define contact based on a force criterion, and we find it more convenient to use a criteria based on the nearest neighbor distance between atoms on the two surfaces. Thus, when the separation between two opposing surface atoms is less than some critical value, contact is defined to occur. However, we have found that neither of these two criteria gives fully satisfactory results. The reason is that if the critical force or the critical distance used to define when contact occurs is determined by fitting the Hertz pressure profile (17) to the numerical data as in Fig. 5 . then the resulting values depend on the radius of curvature of the asperity. For example, for the Hertz contact in Fig. [5 the contact area deduced from the atomistic MD calculation agree with the Hertz theory if we choose the cut-off pressure $p_{\mathrm{c}} \approx 0.7 \mathrm{GPa}$. However, if the radius of curvature of the asperity is 10 times smaller $(R=104 \AA)$ then, for the same penetration, the cutoff would be $p_{\mathrm{c}} \approx 2.5 \mathrm{GPa}$, i.e., more than three times larger. On the other hand randomly rough surfaces have a wide distribution of curvatures and it is not clear how

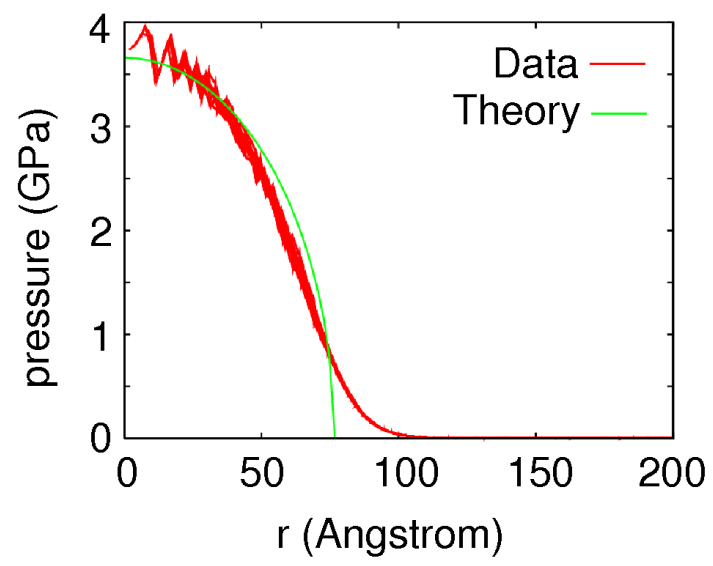

FIG. 5: The pressure in the contact region between a spherical tip and a flat elastic surface. We show the simulation data and the theoretical Hertz result. The spherical tip has the radius of curvature $R=1040 \AA$ and the loading force $4.6 \times 10^{-7} \mathrm{~N}$.

to choose the optimum cut-off distance or force. In this paper we have therefore used another way of determining the contact area which turned out to be more unique. We will now describe this method.

Let us consider the pressure distribution $P(\sigma, \zeta)$ at the interface. For Hertz contact we get the pressure distribution

$$
P(\sigma)=\frac{1}{A_{0}} \int_{A_{0}} d^{2} x \delta(\sigma-\sigma(\mathbf{x}))
$$

Using $\sigma(\mathbf{x})$ from (7) for $r<r_{\mathrm{H}}$ and $\sigma(\mathbf{x})=0$ for $r>r_{\mathrm{H}}$ gives

$$
P(\sigma)=\left(1-\frac{A}{A_{0}}\right) \delta(\sigma)+\frac{2 \sigma}{\sigma_{\mathrm{H}}^{2}} \frac{A}{A_{0}}
$$

where $A=\pi r_{\mathrm{H}}^{2}$ is the Hertz contact area. In Fig. [6] we show the pressure distribution in the contact region between a hard spherical tip and an elastic solid with a flat surface. The red curve shows the simulation data, while the green curve is the theoretical Hertz result obtained by a suitable choice of $A$ in Eq. (9). Note that while the Hertz solution and the atomic MD simulation results agree very well for large pressure, there is a fundamental difference for small pressure. Thus, for the Hertz solution, for small pressure $\sigma \rightarrow 0, P(\sigma) \sim \sigma$, while in the atomistic model $P(\sigma)$ increase monotonically as $\sigma \rightarrow 0$. This difference is due to the long-range interaction between the solid walls in the atomistic model, which is absent in the Hertz model. When the long range wallwall interaction is taken into account the delta function at $\sigma=0$ in the Hertz solution (9) will broaden, resulting in a $P(\sigma)$ which (for the small systems considered here) will decay monotonically with increasing $\sigma$ as observed for the atomistic model. Note that this effect is of exactly 


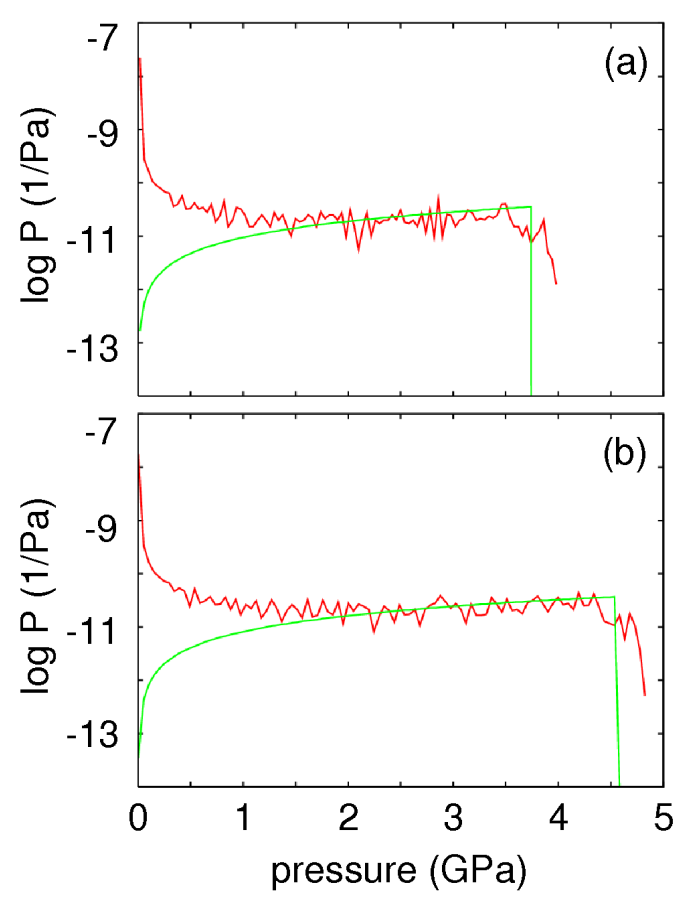

FIG. 6: The pressure distribution in the contact region between a spherical tip and a flat surface. We show the simulation data (red curves) and the theoretical Hertz result (green curves). Loading force in (a) is $4.6 \times 10^{-7} \mathrm{~N}$ and in (b) $7.3 \times 10^{-7} \mathrm{~N}$.

the same origin as the "pressure tail" for $r>r_{\mathrm{H}}$ in Fig. 5

The fact that $P(\sigma, \zeta)$ vanish linearly with $\sigma$ as $\sigma \rightarrow 0$ is an exact result in continuum mechanics with contact interaction (no long range wall-wall interaction), and is valid not just for the Hertz contact case, but holds in general 14]. However, as explained above, this effect will never be observed in the atomistic model if the wall-wall interaction is long-ranged.

Note that the contact area $A$ can be determined directly by fitting the analytical expression for $P(\sigma)$ for the Hertz contact (Eq. (9)) to the numerical MD results for large enough pressures (see Fig. 6). In the present case, for $F_{\mathrm{N}}=4.6 \times 10^{-7} \mathrm{~N}$ (Fig. [6(a)) this gives a contact area $A=\pi r_{\mathrm{H}}^{2}$ which is nearly identical to the one deduced from the fit in Fig. 5. A similar procedure can be used to determine the contact area between randomly rough surfaces using the following analytical expression derived from the contact mechanics theory of Persson (see Eq. (10) below):

$$
P(\sigma, \zeta)=\frac{1}{2(\pi G)^{1 / 2}}\left(e^{-\left(\sigma-\sigma_{0}\right)^{2} / 4 G}-e^{-\left(\sigma+\sigma_{0}\right)^{2} / 4 G}\right),
$$

where $\sigma_{0}$ is the nominal contact stress, and where the fitting parameter $G=G(\zeta)$ can be related to the contact area using Eq. (3). Thus, if $A / A_{0} \ll 1$ we have $G=\left(\sigma_{0}^{2} / \pi\right)\left(A / A_{0}\right)^{-2}$. We have found (see below) that

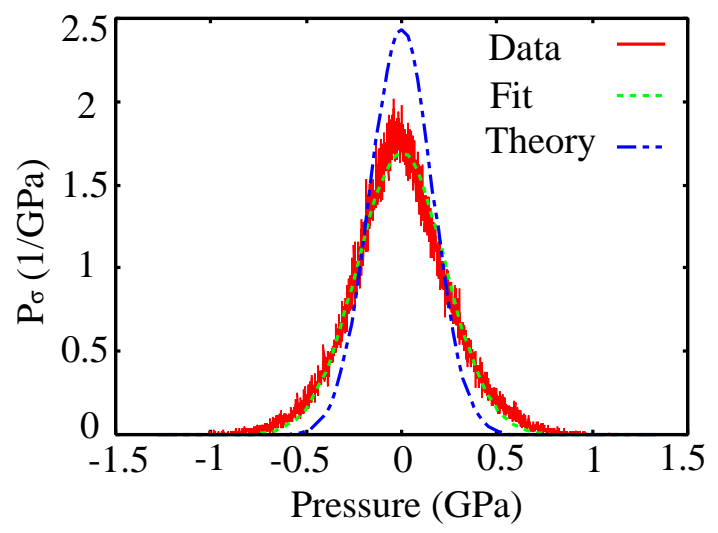

FIG. 7: The normalized pressure distribution $P(\sigma)$ at the interface between an elastic block (elastic modulus $E=$ $0.5 \mathrm{GPa}$ ) with a flat surface and a rigid randomly rough substrate. Because of adhesion complete contact occurs at the interface. The red curve is the simulation result and the green line is the Gaussian fit to the simulation data with the root-mean-square width $\sigma_{\mathrm{rms}}=0.229 \mathrm{GPa}$. The blue line is the theoretical Gaussian distribution obtained using continuum mechanics (see Appendix B). The theoretical rms width $\sigma_{\mathrm{rms}}=0.164 \mathrm{GPa}$.

this expression for $P(\sigma, \zeta)$ can fit the numerical MD data very well (lending support for the accuracy of the Persson theory), and we have used this method to determine the contact area as a function of the squeezing force for randomly rough substrates.

Let us consider the pressure distribution at the interface between a rigid randomly rough substrate and a flat elastic surface when the solids are in complete contact. Complete contact can result either by squeezing the solids together by high enough force, or if the adhesional interaction between the solids is high enough (or the elastic modulus small enough). However, when complete contact occurs the pressure distribution is the same.

For an elastic solid with a flat surface in perfect contact with a hard randomly rough surface, continuum mechanics predict a Gaussian pressure distribution of the form (see Appendix B):

$$
P(\sigma)=\frac{1}{(2 \pi)^{1 / 2} \sigma_{\mathrm{rms}}} e^{-\left(\sigma-\sigma_{0}\right)^{2} / 2 \sigma_{\mathrm{rms}}^{2}}
$$

where the root-mean-square width $\sigma_{\text {rms }}$ is determined by the power spectrum:

$$
\sigma_{\mathrm{rms}}^{2}=\left\langle\sigma^{2}\right\rangle=\frac{\pi}{2} \frac{E^{2}}{\left(1-\nu^{2}\right)^{2}} \int_{q_{0}}^{q_{1}} d q q^{3} C(q)
$$

In Fig. 7 we compare the theoretical pressure distribution (blue curve) with the pressure distribution obtained from the atomistic model for the case where the complete contact results from the adhesive interaction between the solids. The MD data are well fitted by a Gaussian curve, but the width of the curve is slightly larger 


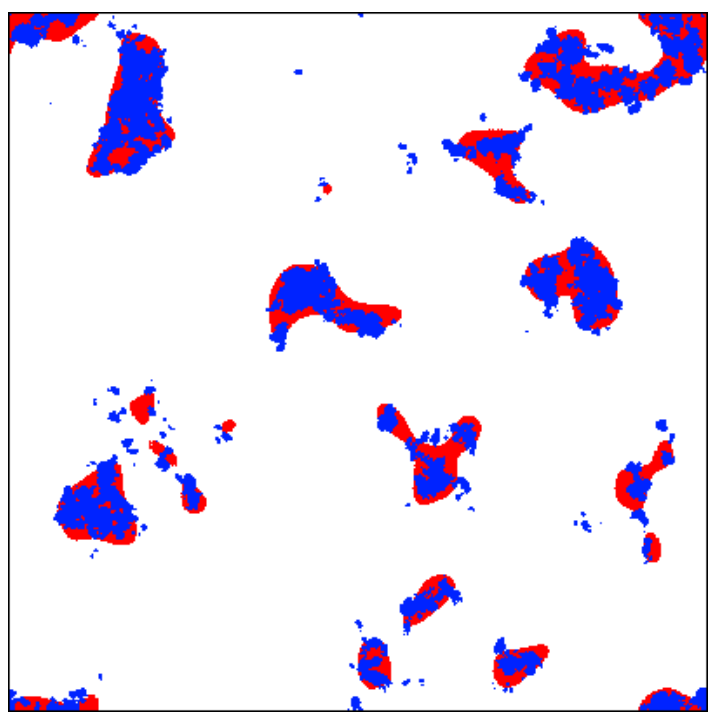

FIG. 8: Contact morphology for two different magnifications. The red color denotes contact regions for the low magnification $\zeta=4$, while the blue color corresponds to the contact regions for the high magnification $\zeta=216$.

than expected from the continuum mechanics theory $\sigma_{\text {rms }}(\mathrm{MD})=0.229 \mathrm{GPa}$ while $\sigma_{\text {rms }}($ theory $)=0.164 \mathrm{GPa}$. The randomly rough surface used in the MD calculation is self affine fractal the whole way down to the atomic distance, and one can therefore not expect the continuum mechanics result for $P(\sigma)$, which assumes "smooth" surface roughness, to agree perfectly with the MD result.

\subsection{Contact mechanics without adhesion}

Here we study contact mechanics without adhesion as obtained with $\alpha=0$ in Eq. (4), corresponding to purely repulsive interaction between the walls. Fig. 8 shows the contact morphologies at different magnifications $\zeta$ for the same load. The red and blue color indicate the contact area at low $(\zeta=4)$ and high $(\zeta=216)$ magnification, respectively. Note that with increasing magnification the contact area decreases, and the boundary line of the contact islands becomes rougher. In Ref. 15] and 16] it has been shown that the statistical properties of the contact regions exhibit power-law scaling behavior. At low magnification $(\zeta=4)$ it looks as if complete contact occurs between the solids at asperity contact regions. However, when the magnification is increased, smaller length scale roughness is detected and it is observed that only partial contact occurs at the asperities. In fact, if there were no short distance cut-off in the surface roughness, the true contact area would eventually vanish. But in reality a short distance cut-off always exists, e.g. the interatomic distance.

Fig. 9 shows the pressure distribution in the contact area for two different magnifications. When we study contact on shorter and shorter length scale, which corresponds to increasing magnification $\zeta$, the pressure distri-

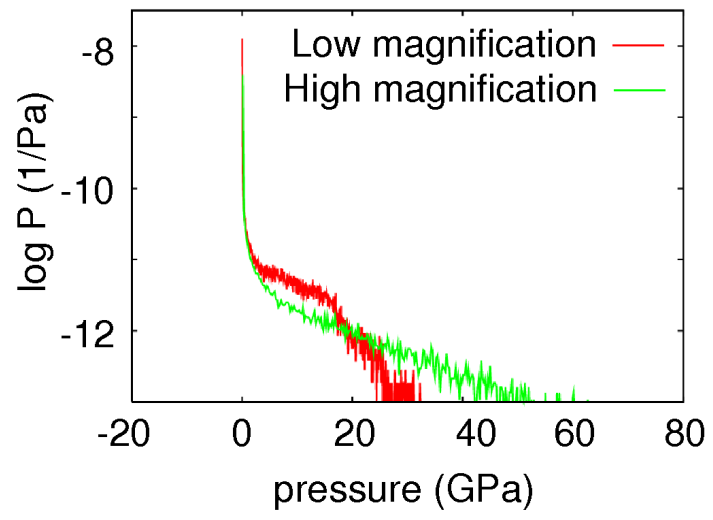

FIG. 9: The pressure distribution in the contact area for two different magnifications. The red line corresponds to the pressure distribution for low magnification $\zeta=4$, while the green line is for high magnification $\zeta=216$.

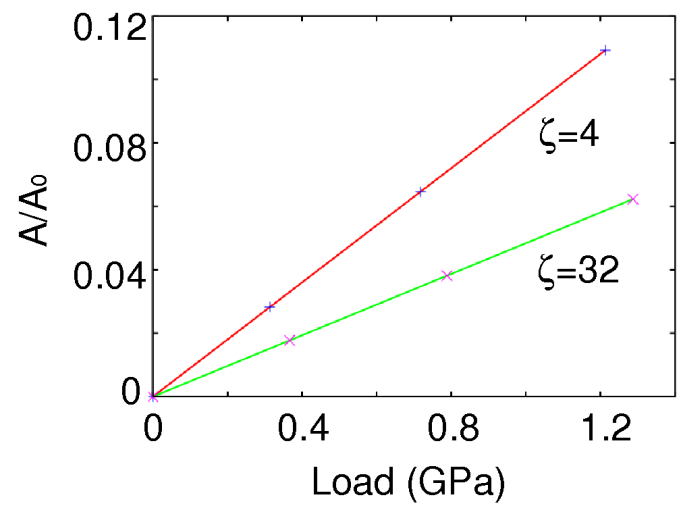

FIG. 10: The relative contact area $A / A_{0}$, as a function of applied stress $F_{\mathrm{N}} / A_{0}$. Results are presented for two different magnifications $\zeta=\lambda_{0} / \lambda=4$ and 32. The fractal dimension is $D_{f}=2.2$.

bution becomes broader and broader.

Fig. [10] shows that the contact area varies (approximately) linearly with the load for the small load at two different magnifications $\zeta=4$ and 32. The contact area was determined as described in Sec. 4.1. by fitting the pressure distribution to a function of the form (10). The pressure distributions and the fitting functions are shown in Fig. 11 and 12 for $\zeta=4$ and 32, respectively. The slope of the lines in Fig. [10 is only a factor 1.14 larger than predicted by the contact theory of Persson (see Sec. $5)$.

In Fig. 13 we show the variation of the contact area with the nominal squeezing pressure for the highest magnification case $\zeta=216$. In this case we have defined contact to occur when the separation between the surfaces is below some critical value $r_{c}=4.3615 \AA$. In contrast to the definition used above, this definition does not give a strict linear dependence of the contact area on the load for small load as found above when the contact area is 


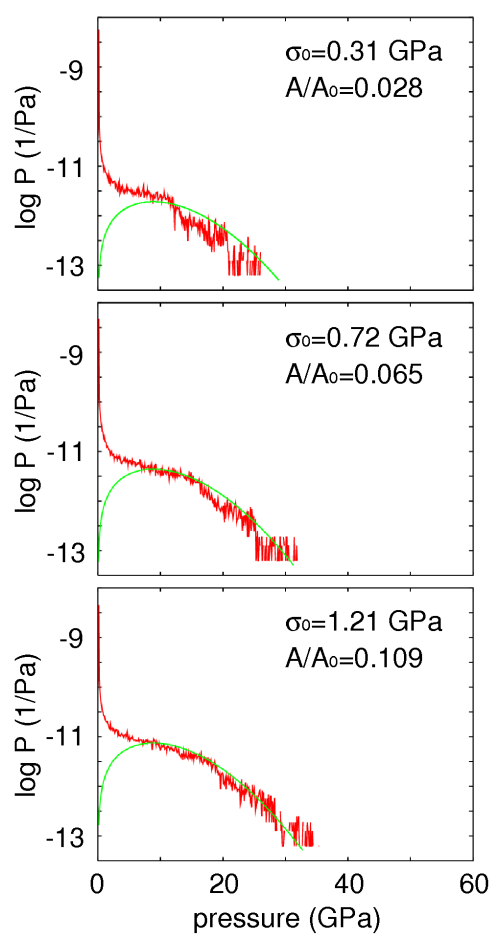

FIG. 11: The stress distribution for $\zeta=4$ for three different nominal pressure.

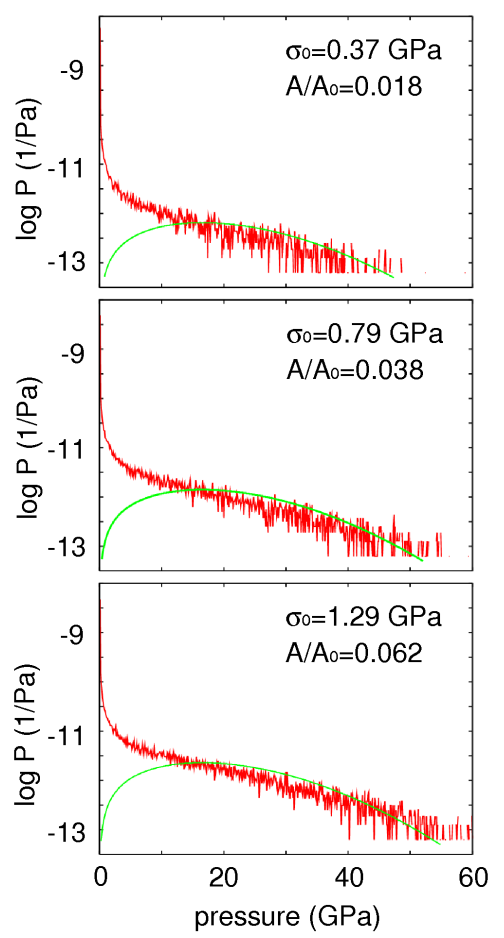

FIG. 12: The stress distribution for $\zeta=32$ for three different nominal pressure.

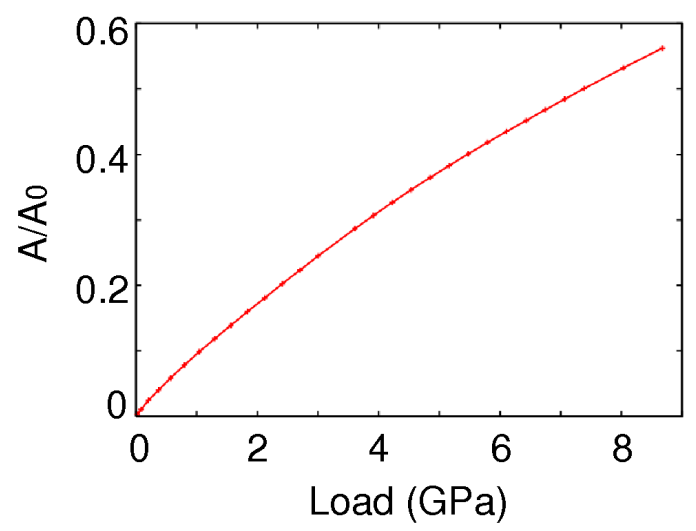

FIG. 13: The relative contact area $A / A_{0}$, as a function of applied stress $F_{\mathrm{N}} / A_{0}$. Results are presented for the highest magnification $\zeta=216$. Contact is defined when the separation between the surfaces is below a critical value. The fractal dimension is $D_{f}=2.2$.

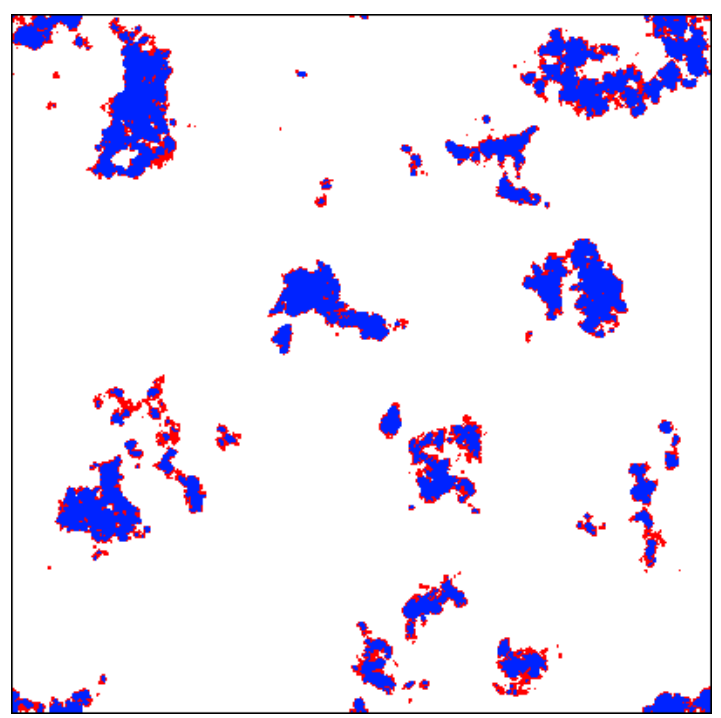

FIG. 14: Contact morphology with adhesion and without adhesion. The blue color region denotes the contact without adhesion. The red color region denote the additional contact area when the adhesional interaction is included.

defined using the stress distribution.

\subsection{Contact mechanics with adhesion}

In this section we include the adhesive interaction i.e. we put $\alpha=1$ in Eq. (4). Fig. 14 presents the contact morphology both with and without the adhesion at the highest magnification $(\zeta=216)$. The regions with blue color denotes the contact area without adhesion. The red color region denotes the additional contact area when adhesion is included. The contact area with adhesion is, of course, larger than that without adhesion since the attractive adhesional interaction will effectively increase the external load 17, 18, 19].

Fig. 15] shows the pressure distribution $P(\sigma, \zeta)$ at high 


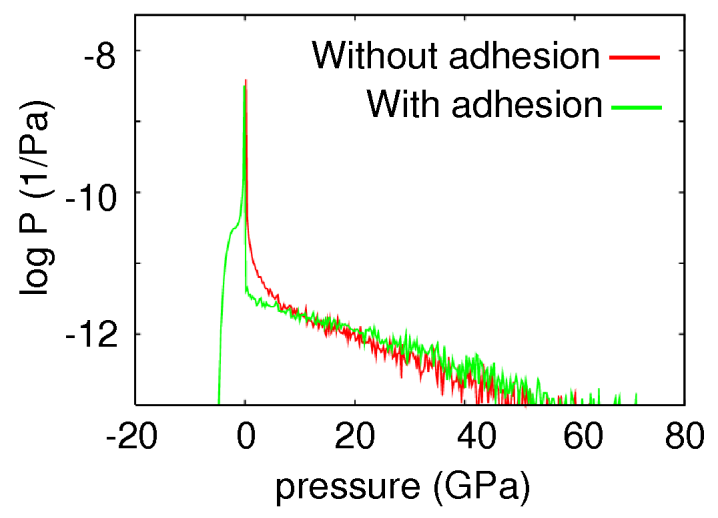

FIG. 15: The pressure distribution with and without adhesion. The red curve denotes the pressure distribution with adhesion while the green curve is without adhesion.

magnification with and without adhesion. When adhesion is neglected (corresponding to the $\alpha=0$ in (4)), the pressure is positive in the contact area and $P(\sigma, \zeta)=0$ for $\sigma<0$. When the adhesive interaction is included, the stress becomes tensile close to the edges of every contact region and $P(\sigma, \zeta)$ is in general finite also for $\sigma<0$.

\section{Discussion}

Several analytical theories, based on continuum mechanics, have been developed to describe the contact between elastic bodies both with and without the adhesional interaction. Here we will compare the results presented above with the predictions of some of these theories.

Persson [4, 5] has developed a contact mechanics theory where the surfaces are studied at different magnification $\zeta=\lambda_{0} / \lambda$, where $\lambda_{0}$ is the roll-off wavelength and $\lambda$ the shortest wavelength roughness which can be observed at the magnification $\zeta$. In this theory [4] the stress distribution $P(\sigma, \zeta)$ at the interface between the block and the substrate has been shown to obey (approximately) a diffusion-like equation where time is replaced by magnification and spatial coordinate by the stress $\sigma$. When the magnification is so small that no atomic structure can be detected, the surface roughness will be smooth (no abrupt or step-like changes in the height profile) and one can then show 14] that in the absence of adhesion $P(0, \zeta)=0$. Using this boundary condition the solution to the diffusion-like equation gives the pressure distribution at the interface $(\sigma>0)$ :

$$
P(\sigma, \zeta)=\frac{1}{2(\pi G)^{1 / 2}}\left(e^{-\left(\sigma-\sigma_{0}\right)^{2} / 4 G}-e^{-\left(\sigma+\sigma_{0}\right)^{2} / 4 G}\right)
$$

where

$$
G=\frac{\pi}{4}\left(\frac{E}{1-\nu^{2}}\right)^{2} \int_{q_{L}}^{\zeta q_{0}} d q q^{3} C(q) .
$$

The relative contact area

$$
\frac{A}{A_{0}}=\int_{0}^{\infty} d \sigma P(\sigma, \zeta)
$$

Substituting (10) into (12) gives after some simplifications

$$
\frac{A}{A_{0}}=\frac{1}{(\pi G)^{1 / 2}} \int_{0}^{\sigma_{0}} d \sigma e^{-\sigma^{2} / 4 G}
$$

Thus, for small nominal squeezing pressure $\sigma_{0} \ll G^{1 / 2}$ we get

$$
\frac{A}{A_{0}}=\frac{\sigma_{0}}{(\pi G)^{1 / 2}} .
$$

Since the squeezing force $F_{\mathrm{N}}=\sigma_{0} A_{0}$ we can also write

$$
A=\kappa \frac{F_{\mathrm{N}}}{E^{*}}\left(\int d^{2} q q^{2} C(q)\right)^{-1 / 2}
$$

where $E^{*}=E /\left(1-\nu^{2}\right)$ and $\kappa=(8 / \pi)^{1 / 2}$. Thus, for small squeezing force $F_{\mathrm{N}}$ the theory predicts a linear dependence of the area of real contact on the load.

For very high squeezing force $\sigma_{0} \gg G^{1 / 2}$ complete contact will occur at the interface. In this case the second term on the rhs in (10) can be neglected, so the pressure distribution is a Gaussian centered at $\sigma_{0}$ and with the root-mean-square width $\sigma_{\mathrm{rms}}=(2 G)^{1 / 2}$. This result is exact (see Appendix B). Thus, the theory of Persson is expected to give a good description of the contact mechanics for all squeezing forces. All other analytical contact mechanics theories are only valid when the squeezing force is so small that the area of real contact is (nearly) proportional to $F_{\mathrm{N}}$. But in many important applications, e.g., in the context of rubber friction and rubber adhesion, the area of real contact for smooth surfaces is often close to the nominal contact area.

The standard theory of Greenwood and Williamson [20] describe the contact between rough surfaces (in the absence of adhesion), where the asperities are approximated by spherical cups with equal radius of curvature but with Gaussian distributed heights. In this theory the area of real contact dependent (slightly) non-linearly on the load for small load, and can therefore not be directly compared with the Persson result (15). Bush et al 21] developed a more general and accurate contact theory. They assumed that the rough surface consists of a mean plane with hills and valleys randomly distributed on it. The summits of these hills are approximated by paraboloids, whose distributions and principal curvatures are obtained from the random precess theory. As a result 
of more random nature of the surface, Bush et al found that at small load the area of contact depends linearly on the load according to (15) but with $\kappa=(2 \pi)^{1 / 2}$. Thus the contact area of Persson's theory is a factor of $2 / \pi$ smaller than that predicted by Bush. Both the theory of Greenwood and Williamson and the theory of Bush et al assume that the asperity contact regions are independent. However, as discussed in [14], for real surfaces (which always have surface roughness on many different length scales) this will never be the case even at a very low nominal contact pressure, which may be the origin of difference of $2 / \pi$ between Persson's theory and Bush's theory.

Hyun et al performed a finite-element analysis of contact between elastic self-affine fractal surfaces [16]. The simulations were done for rough elastic surface contacting a perfectly rigid flat surface. They found that the contact area varies linearly with the load for small load. The factor $\kappa$ was found to be between the results of the Bush and Persson theories for all fractal dimensions $D_{\mathrm{f}}$. For $D_{\mathrm{f}}=2.2$ (corresponding to $H=0.8$ ) they found that $\kappa$ was only $\sim 13 \%$ larger than predicted by the Persson theory.

The red curves in Fig. [16] shows the pressure distribution from the simulations for several different values of the magnification $\zeta=q_{1} / q_{0}=4,8,32$ and 216 , neglecting the adhesion. In the simulations the nominal squeezing pressure $\sigma_{0}=800 \mathrm{MPa}$. The best fit (green curves in Fig. 16) of the pressure distribution (10) to the numerical results is obtained if $G^{-1 / 2}$ is taken to be a factor 1.14 larger than predicted by the Persson theory [Eq. (10)], corresponding to a contact area which is $14 \%$ larger than predicted by the analytical theory, in good agreement with the results obtained by Hyun et al.

Our simulations show that the contact area varies linearly with the load for small load, see Fig. 10] Figs. 10 and [16] show that the slope $\alpha(\zeta)$ of the line $A=\alpha(\zeta) F$ decreases with increasing magnification $\zeta$, as predicted by the analytical theory [14, 15]. Thus, while $A / A_{0}=0.072$ for $\zeta=4$ we get $A / A_{0}=0.038$ for $\zeta=32$, which both are $14 \%$ larger than predicted by Eq. (13).

\section{Summary and conclusion}

In this paper we have developed a Molecular Dynamics multiscale model, which we have used to study the contact between surfaces which are rough on many different length scales. We have studied the contact morphologies both at high and low magnification, with and without adhesion. We have shown that in atomistic models it is a non-trivial problem how to define the area of real contact between two solids. Our study shows that the area of real contact is best defined by studying the interfacial pressure distribution, and fitting it to an analytical expression. The numerical results are consistent with the theoretical results that the contact area varies linearly

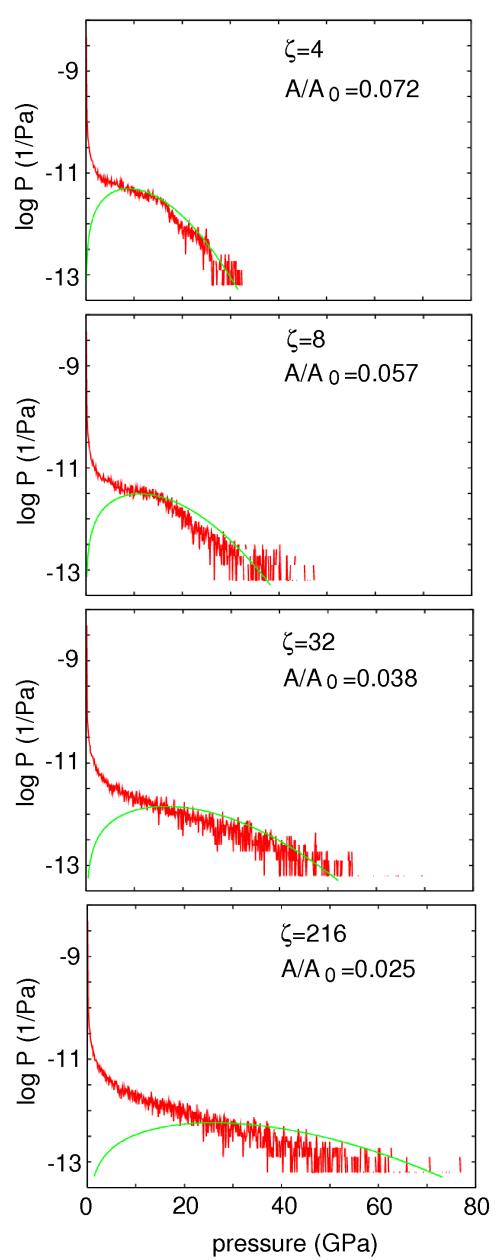

FIG. 16: The pressure distribution at four different magnifications $\zeta=q_{1} / q_{0}=4,8,32$ and 216 for the squeezing pressure $\sigma_{0}=800 \mathrm{MPa}$. The red curves is the pressure distribution obtained from the computer simulation, while the green curves is from the analytical theory assuming that $G^{-1 / 2}$, and hence the relative contact area, is a factor of 1.14 larger than predicted by the analytical theory, Eq. (10).

with the load for small load, where the proportionality constant depends on the magnification $L / \lambda$. For a randomly rough surfaces with the fractal dimension $D_{\mathrm{f}}=2.2$ (which is typical for many real surfaces, e.g., produced by fracture or by blasting with small particles) we have found that for small load (where the contact area is proportional to the load) the numerical study gives an area of atomic contact which is only $\sim 14 \%$ larger than predicted by the analytical theory of Persson. Since the Persson's theory is exact in the limit of complete contact, it is likely that the Persson theory is even better for higher squeezing loads.

Acknowledgments This work was partly sponsored by MIUR FIRB RBAU017S8 R004, FIRB RBAU01LX5H, MIUR COFIN 2003 and PRIN- 

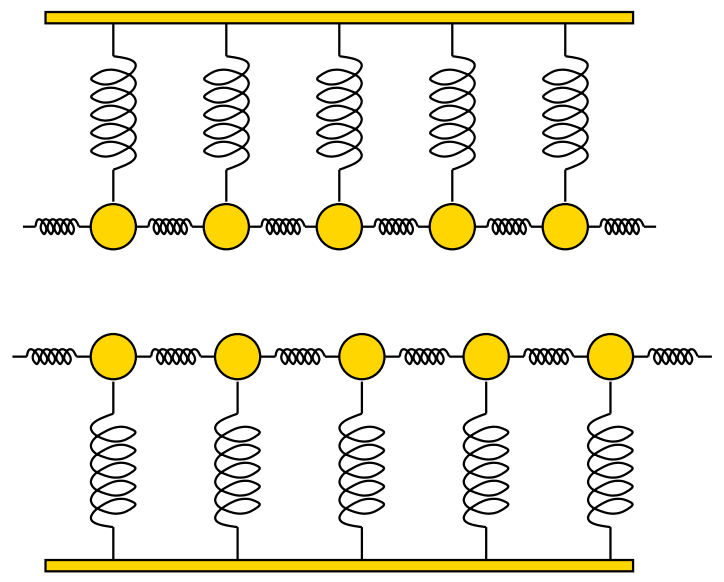

FIG. 17: The model of Persson and Ballone with long range elasticity. Side view.

\section{COFIN2004}

\section{Appendix A: The "smartblock" model for mul- tiscale molecular dynamics}

Here we present a detailed description of the multiscale model implemented in our Molecular Dynamics (MD) simulations. Persson and Ballone 22 introduced a simple and effective model to study the boundary lubrication between elastic walls. For each wall only the outermost layer of atoms was considered. These atoms were able to interact with the lubricant or with the atoms of the other wall with Lennard-Jones potentials. The walls' atoms were connected to a rigid surface through special springs which exert an elastic reaction not only to elongation, but also to lateral bending. The walls' atoms are coupled with their in-plane neighbors with similar springs. It is also possible to use curved elastic walls by connecting the vertical springs to a curved rigid surface rather than a flat surface as in Fig. 17

The model of Persson and Ballone catches two essential features: Firstly the walls are not rigid, they can deform (differently from previous models) and the description takes into account the elastic energy stored during compression or stretching, which is an essential ingredient for the study of the squeeze-out. Secondly both the Young modulus and the shear modulus can be independently tuned via the choice of the elastic constants of the springs.

The model of Persson and Ballone works well when the solid is exposed to uniform shear or uniform elongation or compression. However, when there are spatial variations in the stress at the surface of the solid, for instance when the displacement at the interface comprises short wavelength Fourier components, then the model does not allow a proper description of the elastic deformation field. In particular, when a periodic stress acts on the surface of an elastic solid, the displacement field decays expo- nentially into the solid, and this aspect is absent in the Persson-Ballone model.

The solution to overcome this limitation is straightforward: we explicitly introduce many layers of atoms, placed on the points of a simple cubic lattice, and coupled with springs to their nearest neighbors.

The "springs", as in the previous model, are special, since they can resist to lateral bending. The force due to a vertical spring connecting two consecutive atoms 1 and 2 along the $z$ axis is given by the formulas below, where $a$ is the lattice spacing, that is the equilibrium length of the spring:

$$
F_{x}=-k_{b} \Delta x=-k_{b}\left(x_{2}-x_{1}\right),
$$

$$
F_{y}=-k_{b} \Delta y=-k_{b}\left(y_{2}-y_{1}\right)
$$

$$
F_{z}=-k(\Delta z-a)=-k\left[z_{2}-\left(z_{1}+a\right)\right] .
$$

Analogous formulas hold for the springs parallel to the $y$ and to the $z$ axes. The two elastic constants of the spring, namely $k$ and $k_{b}$, are related to the Young modulus $E$ and the shear modulus $G$ respectively: $k=E a$ and $k_{b}=G a$.

In some circumstances it is useful to simulate quite large and thick samples. Moreover high resolution up to the atomic level is needed in part of the sample, typically at the interface. The solution to avoid excessive computational time is a multiscale approach: high resolution is achieved where it is needed, but a coarse grained description is employed when it is feasible. The coarse graining can happen more times, and to various degrees of resolution, so that a multilevel description of the system comprising many hierarchies is implemented.

The grid structure of the smartblock allows a simple procedure to achieve a multiscale description: groups of atoms can be replaced by single, bigger atoms, and the elastic constants of the springs are redefined to guarantee the same elastic response. In many calculations performed by our group we used to replace a cube of $2 \times 2 \times 2$ particles with a single particle, repeating this merging procedure every two layers. More generally any change of resolution involves merging together a box made of $m_{x} \times m_{y} \times m_{z}$ particles. The three numbers $m_{x}, m_{y}$ and $m_{z}$ are called merging factors along the three axes.

The equilibrium position of the new particle is in the center of mass of the group of particles merged together. Its mass is $m_{x} m_{y} m_{z}$ times the mass of the original particles, so that the density does not change. In fact the masses are only important to study the kinetic, but they do not influence the static equilibrium configuration.

The three merging factors can be chosen independently. The easiest way to calculate the new springs' elastic constants is by considering the merging only along one of the axes. Fig. 18 sketches the case $m_{z}=2$, $m_{x}=m_{y}=1$ (no change of lattice constant along $x$ 


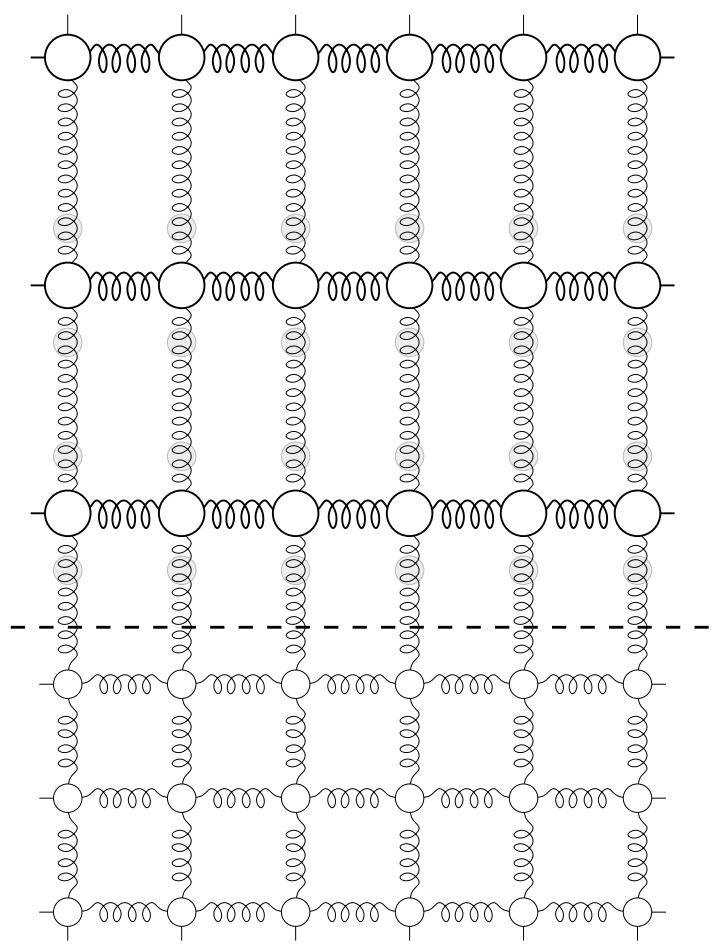

FIG. 18: The grid of particles is coarse grained by replacing two atoms with a single one. Merging factors $m_{x}=m_{y}=1$, $m_{z}=2$. Masses, equilibrium positions and spring constants are changed accordingly.

and $y$ ). Along the direction of merging the new spring constants for elongation and bending are $k^{\prime}=k / m_{z}$ and $k_{b}^{\prime}=k_{b} / m_{z}$ respectively. The longer springs get proportionally smaller elastic constants, as it happens when springs are connected in series. In the two directions orthogonal each spring replaces $m_{z}$ old springs in parallel configuration, so the elastic constants increase proportionally: $k^{\prime}=m_{x} k, k_{b}^{\prime}=m_{x} k_{b}$. Below there is the general formula giving the new elastic constants of the springs along the $z$ axis, with arbitrary merging factors:

$$
k^{\prime}=\frac{m_{x} m_{y}}{m_{z}} k ; \quad k_{b}^{\prime}=\frac{m_{x} m_{y}}{m_{z}} k_{b}
$$

Analogous formulas hold for the springs parallel to the $x$ and $y$ axes.

To get the whole picture we have to characterize the springs at the interface between the two lattices, e.g., the ones crossing the dashed line in Fig. 18 When the merging is in the direction $z$ orthogonal to the interface both elastic constants $k$ and $k_{b}$ get multiplied by the factor $2 /\left(1+m_{z}\right)$. Actually their length is $\frac{1}{2}\left(m_{z}+1\right) a_{z}$, $a_{z}$ being the old lattice constant along $z$. Each of these interface springs can be thought as half a spring of the old grid connected with half a spring of the new grid.

When the merging is along a direction orthogonal to the interface between the two grids, as sketched in Fig. 19] then the spring constants do not change, but

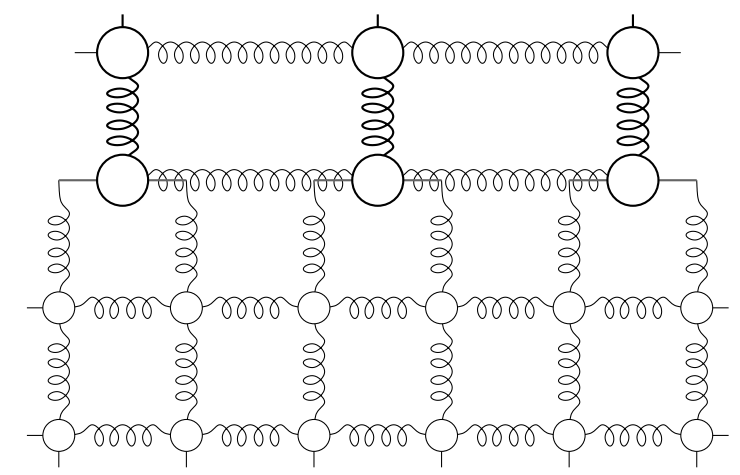

FIG. 19: Change of lattice spacing along a direction parallel to the interface between the two grids.

the forces between the particles are calculated taking into account the in-plane shift between the atoms of the two grids. Each interface particle of the upper lattice interacts with $m_{x} \times m_{y}$ particles of the lower lattice. The equations (A1) and (A2) are modified: $F_{x}=$ $-k_{b}(\Delta x+\mathrm{x}$-shift $), F_{y}=-k_{b}(\Delta x+\mathrm{y}$-shift $)$. The two inplane shifts depend on the pair of particles considered.

\section{Appendix B: Pressure distribution at complete} contact between randomly rough surfaces

Here we calculate the pressure distribution at the interface between two solids in complete contact. We assume that one solid is rigid and randomly rough and the other solid elastic with a flat surface. The pressure distribution

$$
\begin{gathered}
P(\sigma)=\langle\delta(\sigma-\sigma(\mathbf{x}))\rangle=\frac{1}{2 \pi} \int_{-\infty}^{\infty} d \alpha\left\langle e^{i \alpha(\sigma-\sigma(\mathbf{x}))}\right\rangle \\
=\frac{1}{2 \pi} \int_{-\infty}^{\infty} d \alpha e^{i \alpha \sigma} F(\alpha)
\end{gathered}
$$

where

$$
F(\alpha)=\left\langle e^{-i \alpha \sigma(\mathbf{x}))}\right\rangle
$$

where $\sigma(\mathbf{x})$ is the fluctuating pressure at the interface. Next, writing

$$
\begin{aligned}
& \sigma(\mathbf{x})=\int d^{2} q \sigma(\mathbf{q}) e^{i \mathbf{q} \cdot \mathbf{x}} \\
= & \int d^{2} q \frac{E q}{2\left(1-\nu^{2}\right)} h(\mathbf{q}) e^{i \mathbf{q} \cdot \mathbf{x}}
\end{aligned}
$$

where we have used the relation between $\sigma(\mathbf{q})$ and the Fourier transform $h(\mathbf{q})$ of the height profile $h(\mathbf{x})$ derived in Ref. [4], we get

$$
F=\left\langle\exp \left(-i \alpha \int d^{2} q \frac{E q}{2\left(1-\nu^{2}\right)} h(\mathbf{q}) e^{i \mathbf{q} \cdot \mathbf{x}}\right)\right\rangle
$$


Next, using that $h(\mathbf{q})$ are independent random variables we get

$$
F=e^{-\alpha^{2} \xi^{2} / 2}
$$

where

$$
\xi^{2}=\int d^{2} q d^{2} q^{\prime}\left(\frac{E}{2\left(1-\nu^{2}\right)}\right)^{2} q q^{\prime}\left\langle h(\mathbf{q}) h\left(\mathbf{q}^{\prime}\right)\right\rangle e^{i\left(\mathbf{q}+\mathbf{q}^{\prime}\right) \cdot \mathbf{x}}
$$

However (see Ref. [4] )

$$
\left\langle h(\mathbf{q}) h\left(\mathbf{q}^{\prime}\right)\right\rangle=C(q) \delta\left(\mathbf{q}+\mathbf{q}^{\prime}\right)
$$

so that

$$
\xi^{2}=\int d^{2} q\left(\frac{E q}{2\left(1-\nu^{2}\right)}\right)^{2} C(q)
$$

Substituting (B2) in (B1) and performing the $\alpha$-integral and using (B3) gives

$$
P(\sigma)=\frac{1}{(2 \pi)^{1 / 2} \sigma_{\mathrm{rms}}} e^{-\sigma^{2} / 2 \sigma_{\mathrm{rms}}^{2}}
$$

where the root-mean-square width $\sigma_{\text {rms }}$ is determined by the power spectrum:

$$
\sigma_{\mathrm{rms}}^{2}=\left\langle\sigma^{2}\right\rangle=\frac{\pi}{2} \frac{E^{2}}{\left(1-\nu^{2}\right)^{2}} \int_{q_{0}}^{q_{1}} d q q^{3} C(q)
$$

[1] J. Krim, I. Heyvaert, C. Van Haesendonck and Y. Bruynseraede, Phys. Rev. Lett. 70, 57 (1993).

[2] R. Buzio, C. Boragno, F. Biscarini, F.B. de Mongeot and U. Valbusa, Nature Materials 2, 233 (2003).
[3] B.N.J. Persson, O. Albohr, U. Tartaglino, A.I. Volokitin and E. Tosatti, J. Phys. Condens. Matter 17, R1 (2005)

[4] B.N.J. Persson, J. Chem. Phys. 115, 3840 (2001).

[5] B.N.J. Persson, Eur. Phys. J. E8, 385 (2002).

[6] S. Curtarolo and G. Ceder, Phys. Rev. Lett. 88, 255504 (2002)

[7] X.B. Nie, S.Y. Chen, W.N. E and M.O. Robbins, J. Fluid Mech. 500, 55 (2004)

[8] W. E and Z. Huang, Phys. Rev. Lett. 87, 135501 (2001)

[9] W.A. Curtin and R.E. Miller Modelling Simul. Mater. Sci. Eng. 11, R33 (2003)

[10] W. Cai, M. de Koning, V.V. Bulatov and S. Yip, Phys. Rev. Lett. 85, 3213 (2000)

[11] K.L. Johnson, Contact Mechanics (Cambridge University Press, Cambridge, 1985).

[12] J. Israelachvili, Intermolecular and Surface Forces (Academic Press, London, 1992)

[13] B. Luan and M.O. Robbins, Nature 435, 929 (2005).

[14] B.N.J. Persson, F. Bucher, B. Chiaia Phys. Rev. B65, 184106 (2002)

[15] M. Borri-Brunetto, B. Chiaia, M. Ciavarella, Comput. Methods Appl. Mech. Eng. 190, 6053 (2001)

[16] S. Hyun, L. Pei, J.F. Molinari, M.O. Robbins, Phys. Rev. E70, 026117 (2004)

[17] K.L. Johnson, K. Kendall and A.D. Roberts, Proc. R. Soc. A324, 301 (1971).

[18] K. Kendall, Molecular Adhesion and its Applications (New York, Kluwer, 2001).

[19] K.N.G. Fuller and D. Tabor, Proc. R. Soc. A345, 327 (1975).

[20] J.A. Greenwood and J.B.P. Williamson, Proc. Roy. Soc. London, Ser. A295, 300 (1966). See also, J.F. Archard, Proc. Roy. Soc. London, Ser. A243, 190 (1957).

[21] A.W. Bush, R.D. Gibson and T.R. Thomas, Wear 35, 87 (1975); A.W. Bush, R.D. Gibson and G.P. Keogh, Mech. Res. Commun. 3, 169 (1976).

[22] B. N. J. Persson and P. Ballone, J. Chem. Phys. 112, 9524 (2000); Solid State Comm. 115, 599 (2000). 